\title{
Compromiso renal e infección por VIH/SIDA en pacientes atendidos en un hospital pediátrico chileno
}

\author{
Pedro Zambrano O., Ana Chávez P., Ximena Chaparro R., Hugo Castañeda V., \\ Silvia Rakela R., Begoña Corta A., Viola Pinto S. y Elizabeth Eblen Z.
}

\section{Renal compromise in HIV/AIDS in patients attended at a Chilean children hospital}

The aim of this study was to determine the frequency of renal abnormalities in HIV positive children hospitalized in one pediatric hospital in Chile. Method: a cross sectional study was performed during April 2007. Results: A total of 18 patients were evaluated, ten male and eight female ranging in age from 4 to 19 years. The average age at the time of HIV diagnosis and nephrologic evaluations were 2,69 and 10,7 years respectively. All patients had acquired HIV infection by vertical transmission. Urine samples of two children had microalbuminuria; two had monosymptomatic hematuria, and ten had ¿squamous? cells. Hypercalciuria was detected in one patient, renal lithiasis in another and two patients had abnormal renal ultrasonography. All 19 patients had normal blood pressures. Overall 7 patients $(39 \%)$ had a renal abnormality. Conclusions: The relatively high incidence of renal abnormalities in our series support the need for a nation-wide screening program to assess the incidence of renal impairment in pediatric HIV positive patients.

Key words: HIV infection, renal function, HIV associated nephropathy.

Palabras clave: Infección VIH, función renal, nefropatía asociada a VIH.

\section{Introducción}

$\mathrm{L}$ a infección por virus de inmunodeficiencia humana (VIH) ha disminuido dramáticamente su letalidad con la introducción de la terapia antiretroviral (TARV) combinada, cambiando de enfermedad mortal a patología crónica ${ }^{1}$.

Desde los primeros casos pediátricos de infección por VIH en 1983 y $1984^{2-4}$ su incidencia ha aumentado considerablemente en el mundo, siendo África el continente más afectado 5 ; en Chile, a octubre del 2007 se habían reportado 278 niños infectados, 95\% de ellos adquirieron la infección por transmisión vertical (Datos aportados por el Comité Nacional de SIDA Pediátrico).

Una de las complicaciones de esta enfermedad es la nefropatía asociada a infección por VIH (NAVIH); en 1984 se describieron los primeros casos de compromiso renal en pacientes adultos infectados por $\mathrm{VIH}^{6,7}$. La NAVIH puede ser de distintos grados, siendo la injuria directa del virus sobre las células glomerulares y tubulares el principal evento en su patogenia. Existe una disregulación en los podocitos y un aumento en la proliferación de las células tubulares renales, con aumento de factores de crecimiento fibroblástico, por lo que los riñones de estos pacientes están aumentados de tamaño ${ }^{8,9}$.
La presentación clínica puede variar desde proteinuria con síndrome nefrótico a insuficiencia renal crónica terminal. Actualmente no hay tratamiento específico para esta nefropatía $a^{10-12}$.

La incidencia de NAVIH en pediatría es desconocida ya que no se realizan biopsias renales de forma rutinaria, existiendo pocas publicaciones al respecto; Strauss y otros autores han reportado entre 10 y $15 \%$ de compromiso renal en niños, mayoritariamente afroamericanos $^{10-17}$; en Chile no hay datos. Dado que la función renal puede comprometerse durante la evolución de la infección por VIH, en nuestros pacientes se realiza evaluación y seguimiento nefrológico periódico. Los resultados son presentados en esta publicación.

Por otro lado, está demostrado que algunos fármacos anti-retrovirales pueden provocar nefrotoxicidad ${ }^{18}$.

Objetivo: Determinar la prevalencia de alteraciones renales en pacientes pediátricos infectados por VIH, controlados en la Unidad de Infectología del Hospital Dr. Exequiel González Cortés, Santiago de Chile.

\section{Pacientes y Método}

Se realizó un estudio de corte transversal en abril, 2007, de la función renal en todos los pacientes

\author{
Hospital de Niños Dr. Exequiel \\ González Cortés, \\ Santiago, Chile. \\ Unidad de Nefrología (PZO, BCA, \\ VPS) \\ Unidad de Infectología (ACP, SRR) \\ Unidad de Radiología (EEZ) \\ Universidad de Chile, Facultad \\ de Medicina, Santiago, Chile \\ Departamento de Pediatría Sur \\ (XCR, HCV) \\ Recibido: 19 diciembre 2007 \\ Aceptado: 26 agosto 2008
}

Correspondencia a: Pedro Zambrano Ostaíza E-mail: phzambrano@hotmail.com 
Tabla 1. Etapa clínico-inmunológica de la infección por VIH en 18 pacientes pediátricos, según clasificación CDC 1994

\begin{tabular}{|c|c|c|c|c|}
\hline \multicolumn{2}{|c|}{ Categorías inmunológicas } & \multicolumn{3}{|c|}{ Categorías clínicas } \\
\hline & $\mathbf{N}^{*}$ & $A^{* *}$ & $\mathbf{B} * * *$ & $C * * * *$ \\
\hline 1. Sin supresión & 3 & - & 8 & 2 \\
\hline 2. Supresión moderada & 3 & - & - & 1 \\
\hline 3. Supresión grave & - & 1 & - & - \\
\hline
\end{tabular}

${ }^{*} \mathrm{~N}$ : sin síntomas; ${ }^{* *} \mathrm{~A}$ : levemente sintomáticos; $* * * B$ : moderadamente sintomáticos; $\star * * * C$ : gravemente sintomáticos

pediátricos infectados por VIH, en control médico en el hospital. Se analizaron las características demográficas (edad, sexo), vía de contagio, etapa de la enfermedad y TARV; se controló peso, talla y presión arterial (PA). En sangre fueron medidos nitrógeno ureico (NU), creatininemia, calcemia, fosfemia, magnesemia, gases sanguíneos y electrolitos plasmáticos (ELP). Se calculó el aclaramiento de creatinina en base a la fórmula de Schwartz y en los casos en que éste estaba alterado, se midió en orina de 24 horas, considerando un valor normal $>90 \mathrm{ml} / \mathrm{min} / 1,73 \mathrm{~m}^{2}$ superficie corporal ${ }^{19}$. Además, se consignaron hallazgos del examen físico, análisis químico y sedimento de orina, urocultivo, calciuria, microalbuminuria y creatininuria en muestra aislada de orina. Se consideró hipercalciuria al índice calcio/ creatinina mayor a 0,21 en preescolares, escolares y adolescentes, microalbuminuria al índice microalbuminuria/creatininuria mayor a $30 \mathrm{mg} / \mathrm{g}$ de creatinina para todas las edades ${ }^{20,21}$. Si el índice calciuria/creatininuria estaba alterado, se solicitó calciuria de 24 horas. En aquellos pacientes que presentaron proteinuria en el examen de orina completa o índice microalbuminuria/ creatininuria mayor a $30 \mathrm{mg} / \mathrm{g}$ de creatinina, se solicitó proteinuria de 24 horas. En cada paciente se realizó ecotomografía renal y vesical simple. Este protocolo de estudio es parte de la evaluación nefrológica rutinaria en nuestros pacientes con infección por VIH.

\section{Resultados}

En nuestra institución se atendían, al momento del estudio, 19 pacientes; un de ellos fue descartado para este análisis por tener 25 años de edad. De los 18 pacientes evaluados, 10 (55\%) eran de sexo masculino. La edad promedio al diagnóstico de la infección por VIH fue de 2,69 años (rango: 0,08 a 11,4 años). La edad al momento de la evaluación era 10,7 años (rango: 2,3 a 19,0 años). En los 18 pacientes la vía de contagio fue vertical. El tiempo promedio entre el diagnóstico y la evaluación nefrológica fue de ocho años.

El Z peso/edad promedio fue 0,09; el $\mathrm{Z}$ talla/edad fue -0,79; el índice de masa corporal promedio fue 20,3 en pacientes sobre seis años de edad. Todos los pacientes tuvieron cifras de PA normales.

La etapa clínico-epidemiológica de los pacientes, según clasificación CDC $1994^{22}$, se detalla en la Tabla 1 .

En la Tabla 2, se muestran las características de cada paciente relativas a sexo, edad, año del diagnóstico de la infección, etapa clínico-inmunológica, terapia anti-retroviral, recuento de $\mathrm{CD} 4 / \mathrm{mm}^{3}$ y carga viral al momento de la evaluación, y las alteraciones de los exámenes nefrológicos encontradas.

El valor promedio de los resultados de laboratorio se observa en la Tabla 3. Dos pacientes presentaron microalbuminuria, uno tuvo proteinuria de 24 horas elevada y dos hematuria monosintomática. Un paciente presentó litiasis renal (el estudio de litiasis evidenció hipocitraturia) y otro presentó hipercalciuria. En 10 pacientes $(55 \%)$ se encontraron células en el examen de orina, en cinco varones y cuatro mujeres células descamativas y en una niña células epiteliales.

La ecotomografía renal fue anormal en dos pacientes, en uno demostró litiasis renal y en otro se comprobó alteración de la ecogenicidad corticomedular con asimetría renal. Además, en dos niños se observó asimetría renal con ecogenicidad conservada. Uno de los niños había presentado litiasis renal en el período de lactantes, que se resolvió manteniendo el mismo esquema anti-retroviral.

\section{Discusión}

Se han descrito varias anormalidades renales en pacientes con infección por VIH, siendo el hallazgo más frecuente en biopsias la NAVIH; dentro de ella se encuentran la glomeruloesclerosis focal y segmentaria, enfermedad por complejos inmunes y el síndrome hemolítico urémico atípico/púrpura trombocitopénico trombótico. Si bien es cierto que el diagnóstico de la NAVIH se realiza con biopsia renal, hay signos de laboratorio que ayudan a sospecharla, por ejemplo: la presencia de proteinuria o hematuria ${ }^{10,23-25}$. Han y cols, en un estudio realizado en 615 pacientes, encontraron que $38(6 \%)$ tenían algún grado de proteinuria; realizaron biopsia en 23 de ellos encontrando NAVIH en 19 $(83 \%)^{24}$. En nuestra serie, un paciente presentó proteinuria, aunque en rango no nefrótico y en dos pacientes se observó hematuria persistente. Es importante el estudio de la hematuria ya que puede ser una de las formas de presentación de la nefropatía por IgA asociada a $\mathrm{VIH}^{10}$. La microalbuminuria se ha usado clásicamente como predictora de nefropatía en pacientes con diabetes mellitus tipo $\mathrm{I}^{26,27}$; en la actualidad se 


\begin{tabular}{|c|c|c|c|}
\hline$n$ & Sexo & $\begin{array}{l}\text { Edad } \\
\text { (años) }\end{array}$ & $\begin{array}{c}\text { Año de } \\
\text { diagnóstico }\end{array}$ \\
\hline 1 & $\mathrm{~F}$ & 14 & 1993 \\
\hline 2 & $M$ & 15 & 1993 \\
\hline 3 & $M$ & 12 & 1995 \\
\hline 4 & $\mathrm{~F}$ & 19 & 1996 \\
\hline 5 & $\mathrm{~F}$ & 12 & 1996 \\
\hline 6 & $\mathrm{~F}$ & 12 & 1997 \\
\hline 7 & $\mathrm{~F}$ & 10 & 1998 \\
\hline 8 & $M$ & 10 & 1998 \\
\hline 9 & $M$ & 13 & 1998 \\
\hline 10 & $\mathrm{~F}$ & 15 & 1999 \\
\hline 11 & $M$ & 9 & 2000 \\
\hline 12 & $M$ & 11 & 2002 \\
\hline 13 & $M$ & 10 & 2003 \\
\hline 14 & $\mathrm{~F}$ & 6 & 2003 \\
\hline 15 & $M$ & 4 & 2003 \\
\hline 16 & $M$ & 5 & 2004 \\
\hline 17 & $M$ & 4 & 2004 \\
\hline 18 & $F$ & 4 & 2005 \\
\hline
\end{tabular}

\begin{tabular}{lll} 
Etapa* & \multicolumn{1}{c}{ TARV** } & $\begin{array}{c}\text { CD4/mm } \\
\text { (\%) }\end{array}$ \\
C2 & Sin TARV & $236(24)$ \\
B1 & DDI+3TC+RTV & $706(30)$ \\
B1 & Sin TARV & $431(27)$ \\
A3 & AZT+3TC+EFV & $250(13)$ \\
N2 & 3TC+ABA+EFV & $833(27)$ \\
B1 & DDI+3TC+LPV/rtV & $707(25)$ \\
N1 & ZDV+3TC+NVP & $774(35)$ \\
B1 & ABA+EFV+RTV & $592(37)$ \\
N2 & 3TC+EFV+LPV/rtv & $554(21)$ \\
N1 & 3TC+ABA+EFV & $563(28)$ \\
C1 & EFV + LPV/rtV & $783(27)$ \\
N2 & Sin TARV & $367(18)$ \\
C1 & ZDV+DDI+EFV & $1031(37)$ \\
B1 & DDI+3TC+NVP & $1403(39)$ \\
B1 & ZDV+3TC+NVP & $1938(40)$ \\
N1 & Sin TARV & $1106(29)$ \\
B1 & ZDV+3TC+ABA & $1386(36)$ \\
B1 & ZDV+3TC+EFV & $2116(37)$ \\
\hline & &
\end{tabular}

$\begin{gathered}\text { Carga viral copias } \\ \text { ARN/ml /log }\end{gathered}$
$3.400 / 3,53$
ND
$2.300 / 3,36$
$2.300 / 3,36$
ND
ND
ND
ND
ND
ND
ND
$5.400 / 3,73$
ND
ND
ND
$9.700 / 3,98$
ND
ND

Alteraciones encontradas No hay No hay Microalbuminuria No hay No hay No hay Hematuria No hay No hay Hipercalciuria No hay Litiasis-hipocitraturia Microalbuminuria Hematuria No hay No hay No hay Proteinuria

*Etapa clínico inmunológica (CDC 1994); **TARV: terapia antiretroviral. 3TC: lamivudina; ABA: abacavir; RTV: ritonavir; DDI: didanosina; EFV: efavirenz; LPV: lopinavir ZDV: zidovudina; NVP: nevirapina; ${ }^{* * *} \mathrm{ND}$ : no detectable

utiliza en otras enfermedades sistémicas como marcador de hiperfiltración y daño renal inicial ${ }^{28-30}$. Han y cols, encontraron que 7 de 90 pacientes $(8 \%)$ portadores de VIH presentaban microalbuminuria; seis $(86 \%)$ de ellos tuvieron biopsias compatibles con $\mathrm{NAVIH}^{24}$; en nuestra casuística se demostró microalbuminuria en dos pacientes. Todos los pacientes tuvieron PA normal, hecho concordante con lo publicado en la literatura médica ${ }^{25,31}$ y NU, creatininemia, calcemia, fosfemia, gases sanguíneos y ELP dentro de límites normales; estudios publicados muestran alteraciones de estos parámetros en pacientes con infección por $\mathrm{VIH}^{25}$.

Llama la atención el número de pacientes con células descamativas y epiteliales en el examen microscópico de orina en nuestra casuística; este hallazgo es de difícil interpretación. Ray PE y cols, encontraron células epiteliales (escamosas, transicionales) y células del epitelio tubular renal en orina de pacientes con infección por VIH, y comprobaron que estas células no son capaces de transmitir el virus ${ }^{32,33}$.

Varios estudios muestran que recuentos de CD4 bajos tienen mayor relación con NAVIH, sobre todo si hay co-existencia con microalbuminuria ${ }^{24,31,34}$. En nues-
Tabla 3. Valores promedio de exámenes de laboratorio en 18 pacientes pediátricos con infección por ViH

\begin{tabular}{|lc|}
\hline Parámetro & Promedio \\
\hline Nitrógeno ureico & $10,84 \mathrm{mg} / \mathrm{dL}$ \\
\hline Creatininemia & $0,46 \mathrm{mg} / \mathrm{dL}$ \\
\hline Calcemia & $9,37 \mathrm{mg} / \mathrm{dL}$ \\
\hline Fosfemia & $5,1 \mathrm{mg} / \mathrm{dL}$ \\
\hline Magnesemia & $0,81 \mathrm{mg} / \mathrm{dL}$ \\
\hline $\mathrm{pH}$ & 7,34 \\
\hline Exceso de base & $-0,8$ \\
\hline Bicarbonato - & $25,3 \mathrm{mEq} / \mathrm{L}$ \\
\hline Calciuria/creatininuria & $0,11 \mathrm{mg} / \mathrm{dL}$ \\
\hline Microalbuminuria/creatininuria & $15 \mathrm{mg} / \mathrm{g}$ de creatinina \\
\hline CD4/mm 3 (\%) & $869(29)$ \\
\hline
\end{tabular}

tra experiencia, cinco pacientes presentaron recuentos de CD4 en rangos de inmunosupresión moderada o grave, con carga viral detectable; sólo uno de ellos presentó litiasis renal y los otros cuatro tuvieron estudio normal. 
Se ha descrito, en pacientes portadores de NAVIH, riñones aumentados de tamaño para la talla o hiperecogénicos, lo que puede observarse en estadios tempranos o tardíos de la enfermedad ${ }^{25}$. En nuestra serie, dos pacientes presentaron asimetría renal; no sabemos si como una variante anatómica o como parte de una NAVIH no diagnosticada, y un paciente tuvo alteración de la ecogenicidad cortico-medular (paciente con microalbuminuria).

No hay tratamiento específico para la NAVIH, la terapia anti-retroviral ha demostrado mejorar la función renal. Actualmente se utilizan inhibidores de la enzima convertidora de angiotensina II como protector renal; además se usan corticosteroides sistémi$\cos ^{11,25}$.

La Sociedad Americana de Enfermedades Infecciosas (IDSA) recomienda realizar evaluación semestral con NU, creatininemia, ELP, examen químico y microscópico de orina, estudio para proteinuria y además, biopsia renal a pacientes con proteinuria, deterioro de la función renal, insuficiencia renal aguda de etiología no precisada y síndrome nefrítico. Sugiere además, evaluar la necesidad de efectuar biopsia en pacientes con recuentos bajos de CD4 y carga viral elevada ${ }^{25}$; de acuerdo a nuestros resultados y a las publicaciones actuales, sugerimos además realizar estudio para detectar microalbuminuria.

Estos resultados hacen necesario plantear un tamizaje como protocolo nacional para evaluar las altera- ciones de laboratorio y posteriormente realizar biopsia renal a aquellos con exámenes alterados para determinar la incidencia de NAVIH en nuestros pacientes, ya que su manejo oportuno puede retardar el daño renal.

\section{Resumen}

El objetivo de este estudio fue evaluar la función renal de pacientes infectados con virus de inmunodeficiencia humana (VIH) que se controlan en un hospital pediátrico chileno. Método: estudio de corte transversal. Resultados: Se evaluaron 18 pacientes, 10 varones y 8 mujeres; edad: entre 4 y 19 años, la edad promedio al diagnóstico de la infección por VIH y al momento de la evaluación fue 2,69 y 10,7 años, respectivamente. Todos nuestros pacientes adquirieron la infección vía vertical. Dos presentaron microalbuminuria y dos hematuria monosintomática. En 10 (55\%) se encontraron células descamativas, en uno hipercalciuria y en otro litiasis renal. Todos tuvieron presión arterial normal. La ecotomografía renal fue anormal en dos. Se han descrito varias anormalidades renales en pacientes con infección por VIH; en nuestro estudio, 7 pacientes (39\%) tuvieron alteraciones en los exámenes de laboratorio. Conclusión: La alta frecuencia de afectación renal encontrada en pacientes pediátricos con infección por VIH hace necesario plantear un tamizaje nacional para determinar la incidencia de nefropatía asociada en nuestros pacientes.

\section{Referencias}

1.- Wolff M, Beltrán C. Programa de acceso ampliado a terapia antiretroviral en Chile, Plan Auge y cohorte chilena de SIDA: Tres iniciativas complementarias. Rev Chil Infect 2004; 21: 281-4.

2.- Oleske J, Minnefor A, Cooper R, Thomas K, Cruz A de la, Ahdieh H, et al. Immune deficiency syndrome in children. JAMA 1983; 249: 2345-9.

3.- Rubinstein A, Sicklick M, Guota A, Bernstein L, Klein N, Rubinstein E, et al. Acquired immunodeficiency with reversed T4/T8 ratios in infants born to promiscuous and drug-addicted mothers. JAMA 1983; 249: 2350-6.

4.- Cowan M J, Hellman D, Chudwin D, Wara D W, Chan R S, Ammann A J. Maternal transmission of acquired immune deficiency syndrome. Pediatrics 1984; 73: 382-6.

5.- No author listed. The global HIV and AIDS epidemic. MMWR Morbid Mortal Wkly Rep 2001; 50: 439.
6.- Rao T K, Filippone E J, Nicastri A D, Landesman S H, Frank S, Chen C K, et al. Associated focal and segmental glomerulosclerosis in the acquired immunodeficiency syndrome. N Engl J Med 1984; 310: 669-73.

7.- Pardo V, Aldana M, Colton R M, Fischl M A, Jaffe D, Moskowitz L, et al. Glomerular lession in the acquired immunodeficiency syndrome. Ann Intern Med 1984; 101: 429-34.

8.- Weiner J, Goodman J, Kimmel P. The HIVassociated renal disease: Current insight into pathogenesis and treatment. Kidney Int 2004; 63: 1618-31.

9.- Ray P E, Xu L, Rakusan T, Liu X-H. A 20year history of childhood HIV-associated nephropathy. Pediatr Nephrol 2004;19: 1075-92.

10.- Turner M E, Kher K, Rakusan T, D’Angelo L M, Kapur S, Selby D, et al. Atypical hemolytic-uremic syndrome in HIV-1 infected children. Pediatr Nephrol 1997; 11: 161-3.

11.- Ray P E, Rakusan T, Loechelt B J, Selby D
M, Liu X H, Chandra R S. Human immunodeficiency virus (HIV)-associated nephropathy in children from the Washington D.C. area: 12 years' experience. Semin Nephrol 1998; 18: 396-405

12.- Balow J. Nephropathy in the context of HIV infection. Kidney Int 2005; 67: 1632-33.

13.- Connor E, Gupta S, Joshi V, DiCarlo F, Offenberger J, Minnefor A, et al. Acquired immunodeficiency syndrome-associated renal disease in children. J Pediatr 1998; 113: 39-44.

14.- Strauss J, Abitol C, Zilleruelo G, Scott G, Paredes A, Malaga S, et al. Renal disease in children with acquired immunodeficiency syndrome. N Engl J Med 1989; 321: 625-30.

15.- Tarshish P. Approach to the diagnosis and management of HIV-associated nephropathy. J Pediatr 1991; 119: S50-56

16.- Joshi V V. Pathology of childhood AIDS. Pediatr Clin North Am 1991; 84: 11-13.

17.- Ingulli E, Tejani A, Fikrig S, Nicastri A, Chen C K, Pomrantz A. Nephrotic syndrome associated with acquired 
immunodeficiency syndrome in children. J Pediatr 1991; 119: 710-6.

18.- Said S M, Nasr S H, Markowitz G S, D'Agati V D. Nephrotoxicity of antiretroviral therapy in an HIV-infected patient. Kidney Int 2007; 71: 1071-5.

19.- National Kidney Foundation. K/DOQI clinical practice guidelines for chronic kidney disease: evaluation, classification, and stratification. Am J Kidney Dis 2002 Feb; 39 (2 Suppl 1): S1-266.

20.- Gillespie S, Stapleton B. Nephrolithiasis in children. Pediatrics Rev 2004; 21: 131-9.

21.- Mattix H, Hsu Ch-Y, Shaykevich S, Curhan G. Use of the albumin/creatinine ratio to detect microalbuminuria: Implications of sex and race. J Am Soc Nephrol 2002; 13: 1034-9.

22.- Wu E. Infección por virus de inmunodeficiencia humana en la infancia. Rev Chil Infect 2007; 24: 276-83.

23.- Turner M E, Kher K, Rakusan T, D'Angelo L, Kapur S, Selby D, et al. Atypical hemolytic uremic syndrome in human immunodeficiency virus-1-infected children. Pediatr Nephrol 1997; 11: 161-3.

24.- Han T M, Naicker S, Ramdial P K, Assounga A G. A cross-sectional study of HIV-seropositive patients with varying degrees of proteinuria in South Africa. Kidney Int 2006; 69: 2243-50.

25.- Gupta S, Eustace J, Winston J, Boydstun I, Ahuja T, Rodríguez R, et al. Guidelines for the management of chronic kidney disease in HIV-infected patients: Recommendations of the HIV Medicine Association of the Infectious Diseases Society of America. Clin Infect Dis 2005; 40: 1559-85.

26.- Dahlquist G, Stattin E L, Rudberg S. Urinary albumin excretion rate and glomerular filtration rate in the prediction of diabetic nephropathy; a long-term followup study of childhood onset type1 diabetic patients. Nephrol Dial Transplant 2001; 16: 1382-6.

27. - Frazer F L, Palmer L J, Clarey A, Thonell S, Byrne G C. Relationship between renal volume and increased albumin excretion rates in children and adolescents with diabetes mellitus. J Pediatr Endocrinol Metab 2001; 14: 875-81.

28.- Tam L S, Li E K, Benzie I, Lam C, Arumanayagam M, Chung W Y, et al. Metabolic abnormalities associated with microalbuminuria in systemic lupus erythematosus. Rheumatology 2001; 40: 1193-4.

29.- Bianchi S, Bigazzi R, Campese V M. Microalbuminuria in essential hypertension: significance, pathophysiology and therapeutic implications. Am J Kidney Dis 1999; 34: 973-5.

30.- Garg A, Clark W, Salvadori M, Macnab J, Suri R, Haynes B, et al. Microalbuminuria three years after recovery from Escherichia coli $\mathrm{O} 157$ hemolytic uremic syndrome due to municipal water contamination. Kidney Int 2005; 67: 1476-82.

31.- Szczech L, Gange S, Van der Horst C, Bartlett J, Young M, Cohen M, et al. Predictors of proteinuria and renal failure among women with HIV infection. Kidney Int 2002; 61: 195-202.

32.- Ray P E, Liu X H, Xu L, Rakusan T. Accumulation of bFGF in children with HIV-1 associated hemolytic uremic syndrome. Pediatr Nephrol 1999; 13: 586-93.

33.- Ray P E, Liu X H, Henry D, Dye L, Xu L, Orenstein $\mathrm{J} \mathrm{M}$, et al. Infection of human primary renal epithelial cells with HIV-1 from children with HIV-associated nephropathy. Kidney Int 1998; 53: 1217-29.

34.- Laradi A, Mallet A, Beaufils H, Allouache M, Martínez F. HIV-associated nephropathy: Outcome and prognosis factors. J Am Soc Nephrol 1998; 9: 2327-35. 